\title{
La incertidumbre climática y el dilema energético colombiano
}

\author{
(1) Camilo Montes
}

Universidad del Norte, Barranquilla, Colombia

Artículo de posesión para el ingreso como miembro correspondiente a la

Academia Colombiana de Ciencias Exactas, Físicas y Naturales el 22 de noviembre de 2018

\begin{abstract}
Resumen
El fenómeno de El Niño en Colombia está marcado por un déficit de precipitaciones precisamente donde se localizan la mayor parte de las hidroeléctricas del país. Si el clima del Plioceno, hace $\sim 2,6$ a 5,3 millones de años, es análogo al que podríamos esperar durante este siglo, el déficit en las precipitaciones afectaría la generación hidroeléctrica en Colombia. El respaldo de la generación hídroeléctrica en Colombia es el gas. Las reservas de gas y petróleo en nuestro país están agotándose y nuestra ventana de autoabastecimiento está cercana a su fin. La combinación de estos factores hace que la generación eléctrica colombiana sea muy vulnerable a los fenómenos climáticos que implican déficit en las precipitaciones. El calentamiento global, producto principalmente de la quema de carbón, petróleo y gas, podría llevarnos a una situación en la que, paradójicamente, la única alternativa para que el país tenga independencia y seguridad energética sea el carbón. C 2018. Acad. Colomb. Cienc. Ex. Fis. Nat.
\end{abstract}

Palabras clave: El Niño; El Niño - Oscilación Meridional (ENOM); Electricidad; Carbón; Gas; Cambio climático; Colombia.

\section{Climatic uncertainty and the Colombian energy dilema}

\begin{abstract}
The El Niño phenomenon in Colombia is characterized by a rainfall deficit precisely where most of the country's hydroelectric plants are located. Climatic conditions of the Pliocene, $\sim 2.6$ to 5.3 million years ago, a good analogy for the changing global climate, suggest that rainfall deficits would occur in Colombia, negatively impacting hydroelectric generation. The backup for hydroelectric generation in Colombia is gas. Gas and oil reserves in our country are running out and our self-supply window is nearing its end. The combination of these factors make Colombia's electricity generation highly vulnerable particularly to climatic conditions where rainfall deficits exist. Global warming caused by the burning of coal, oil and gas, could bring a scenario where, paradoxically, the only alternative for the country to have independence and energy security would be coal. (C) 2018. Acad. Colomb. Cienc. Ex. Fis. Nat.
\end{abstract}

Key words: El Niño; El Niño Southern Oscillation (ENSO); Electricity; Coal; Gas; Climate change; Colombia.

\section{Introducción}

Más de dos terceras partes de la generación eléctrica colombiana dependen de un predecible régimen de lluvias que llena los embalses entre abril-mayo y septiembre-noviembre, con una regularidad solo interrumpida por el fenómeno de El Niño. No sabemos si este fenómeno, actualmente con una periodicidad subdecadal, podría volverse más frecuente o más grave como resultado del cambio climático. La generación térmica, respaldo del sistema interconectado colombiano, funciona fundamentalmente a partir de gas y combustibles líquidos, con capacidad de generar menos de la mitad del consumo del país. Colombia, sin embargo, se está quedando sin gas y sin petróleo. La inversión en investigación en energías renovables es prácticamente nula. Aún así, el congreso acaba de ratificar el tratado de las partes adoptado en la XXI Conferencia sobre Cambio Climático (COP21, Naciones Unidas, 2015), que aunque bien intencionado, limita nuestras emisiones de gases de efecto invernadero (http://www.minambiente.gov.co/index.php/ noticias/2957-congreso-aprueba-proyecto-de-ley-pararatificar-acuerdo-de-paris).

El consenso alcanzado en París fijó una meta de calentamiento global de $2^{\circ} \mathrm{C}$ o menos (con $1,5{ }^{\circ} \mathrm{C}$ como meta preferida) y $20 \mathrm{~cm}$ de ascenso de nivel del mar con respecto a niveles preindustriales para finales del siglo XXI. Estas metas eran ya extremadamente ambiciosas en el momento

\footnotetext{
Correspondencia:

Camilo Montes, montes.camilo@gmail.com

Recibido: 14 de mayo de 2018

Aceptado: 29 de agosto de 2018

Editor: Carlos Alberto Vargas
} 
de la firma del acuerdo en el 2015. Las emisiones globales de gases de efecto invernadero continuaron creciendo desde la firma en París y, recientemente, el mayor emisor acumulativo de gases de efecto invernadero (Estados Unidos, con el $29,3 \%$ de las emisiones entre 1850 y 2002, Baumert, et al., 2005), se retiró de dicho acuerdo (Tollefson, 2017). Algunos modelos que analizan las tendencias de decarbonización sugieren que en el 2018 debimos haber tenido crecimiento nulo en emisiones de $\mathrm{CO}_{2}$ con respecto a los niveles del 2010 (Rogelj, et al., 2015). Dado que la reducción no ocurrió, y es muy dudoso que ocurra en el siguiente lustro, la ventana de oportunidad para alcanzar las metas establecidas para final de siglo parece ya cerrada. Así haya cálculos más optimistas (Millar, et al., 2017), más que limitar las emisiones, la alternativa de Colombia parece limitarse a la adaptación a los cambios venideros en un planeta con temperaturas promedio $2{ }^{\circ} \mathrm{C}$ más cálidas, más aun teniendo en cuenta que aquellos países que no nos beneficiamos de la abundante energía (cuyo subproducto son las emisiones de los últimos 200 años) estamos siendo afectados por los cambios de manera más intensa (Bathiany, et al., 2018) dada la fragilidad general de su infraestructura (Kreft, et al., 2015). La política consignada en el documento COP21 de Colombia, que es, de facto, la hoja de ruta energética del gobierno, parece desligada de la realidad geológica colombiana e insensible a los posibles escenarios del cambio climático.

Si Colombia pretende seguir un modelo de desarrollo como el europeo y el norteamericano, requiere mucha energía confiable y barata. El acuerdo de las partes COP21 de París - aunque no vinculante - nos pone como sociedad en una encrucijada energética. Dado que el desarrollo, entendido como desarrollo industrial, requiere tanta energía (Woodall, 2011), esta debe ser barata de modo que la industria pueda ser competitiva en el escenario global. En este ensayo exploro alternativas para una hoja de ruta energética a corto y mediano plazo enraizada en la realidad geológica nacional y teniendo en cuenta un posible escenario de cambio climático. Ignoro a propósito los efectos de estas alternativas en el clima del planeta porque Colombia es, y lo ha sido siempre, un contribuidor apenas marginal de las emisiones globales de gases de efecto invernadero (con apenas un 0,46\% de las emisiones globales, Naciones Unidas, 2015). El análisis que presento está sustentado en datos concretos sobre los recursos que tenemos en abundancia, como el carbón, sobre aquellos más escasos, como el petróleo y el gas, y sobre los que podrían escasear en un escenario de cambio climático, como el agua. La solidez o debilidad energética del país depende de las interacciones entre los posibles efectos del cambio climático — como un fenómeno de El Niño más frecuente o intenso- y la naturaleza de la matriz energética que decidamos implementar en las siguientes décadas. Muestro aquí que una continuada dependencia de la energía hidroeléctrica y de la generación por gas es suceptible de multiplicar los efectos negativos del cambio climático en nuestro país.
Es la incertidumbre acerca de la naturaleza y magnitud de los cambios climáticos la que lógicamente nos indica que la ruta más segura para suministrarle energía a nuestra sociedad, a falta de mejores alternativas, es el carbón. Enfatizo que la ruta preferida sería la de la disminución del consumo y las energías renovables, pero como muestro en esta contribución, estas requieren décadas de inversión, construcción de experiencia e investigación. Colombia no ha desarrollado ninguna de las anteriores, y el cambio climático podría, sí, avanzar más rápido que la renovación energética colombiana.

\section{El fenómeno de El Niño}

El fenómeno de El Niño y la oscilación meridional (ENOM) es la fluctuación climática más importante del planeta. En condiciones normales, en el océano Pacífico los vientos alisios empujan agua cálida y superficial hacia el occidente, favoreciendo el afloramiento de aguas profundas y frías a lo largo de la costa occidental suramericana. El contraste de temperaturas superficiales del agua $\left(\sim 4^{\circ} \mathrm{C}\right)$ refuerza el contraste de presiones atmósfericas de este a oeste, intensificando aun más los alisios. Durante un año de El Niño, sin embargo, los alisios se debilitan y la presión atmosférica aumenta en el oeste y disminuye en el este. Esto hace que en un año de El Niño los alisios debilitados y el calentamiento de aguas superficiales en el ecuador se refuercen uno a otro llevando a que una "piscina" de agua cálida se estacione en el normalmente frío Pacífico oriental (Collins, et al., 2010). Durante un año así ocurren muchos cambios, incluidos los que se producen en la frecuencia de los huracanes en el Atlántico (Bove, et al., 1998; Donnelly \& Woodruff, 2007), en las precipitaciones en los trópicos y en los sistemas de monzones (Dai \& Wigley, 2000), en las temperaturas en Canadá y las precipitaciones en Norteamérica (Ropelewski \& Halpert, 1986), entre muchas otros, los cuales afectan los patrones climáticos en todo el planeta. El fenómeno de El Niño es global, con conexiones más o menos intensas en todo el planeta, y, naturalmente, en el norte de los Andes.

El Niño en épocas glaciales. Una forma de tratar de entender la variabilidad de El Niño es estudiar los registros paleoclimáticos de los últimos cientos a miles de años. A escalas de observación de milenios, sin embargo, no parece haber una correlación directa y sencilla entre la recurrencia e intensidad del ENOM con un incremento o disminución en las temperaturas globales. Los registros de corales en el Pacífico tropical sugieren que la actividad más intensa del ENOM ocurrió precisamente en el siglo XVII durante la fría "pequeña edad de hielo" (Cobb, et al., 2003), así como después de los "inviernos" producidos por actividad volcánica (Adams, et al., 2003). En contraste, los registros de inundación costa afuera en Perú (Rein, et al., 2005) documentan algunas correlaciones positivas entre una mayor actividad e intensidad del ENOM y mayores temperaturas globales durante los últimos 17.000 años. Estos estudios sugieren una contemporaneidad y, posiblemente 
una relación de causalidad entre la destrucción de la última masa glacial de Norteamérica (Laurentina) y el inicio del ENOM en Perú. Una compilación de registros globales desde 1.525, sugiere sin embargo que el siglo XX ha tenido una actividad más intensa de El Niño, con 12 de los 37 eventos considerados como fuertes o extremos (Gergis \& Fowler, 2009). En esta misma compilación se reporta que los eventos más largos de El Niño han llegado a durar hasta siete años (1900-1906 y 1718-1724). Así las cosas, algunos registros climatológicos en escalas de siglos y milenios parecieran entregar resultados ambiguos acerca de la correlación entre la intensidad y la frecuencia del ENOM y las temperaturas globales. Otros, sin embargo, parecieran sugerir que las temperaturas atmosféricas más altas se correlacionan positivamente con eventos más pronunciados o prolongados de El Niño.

Predicciones computacionales del ENOM. Otra forma de intentar entender el ENOM es mediante modelos computacionales globales. En ellos se acopla el comportamiento de la atmósfera y los océanos, intentando reproducir $\mathrm{y}$, eventualmente, predecir el clima planetario. Para ello se divide el globo en pequeñas celdas donde se calculan variables climáticas a partir de las ecuaciones que describen los comportamientos físicos de la atmósfera y los océanos. Aunque aún lejos de reproducir los detalles del ENOM, estos modelos parecen sugerir que un incremento en la temperatura planetaria generaría un ENOM más intenso y frecuente (Timmermann, et al., 1999; Yeh, et al., 2009; Cai, et al., 2014; Cai, et al., 2015), incluso si es diferente al que se produjo en el siglo XX. Sin embargo, las altísimas complejidades de los modelamientos numéricos, las parametrizaciones necesarias y las interacciones entre variables todavía constituyen una barrera en la predicción climática de los siguientes 100 años. Existen modelos que, simulando las mismas interacciones, sugieren exactamente lo contrario (van Oldenborgh, et al., 2005). En un experimento para verificar la veracidad de las predicciones, se tomaron los aumentos de las temperaturas planetarias de múltiples modelos de los 20 años anteriores y se compararon con localidades en las que se habían registrado instrumentalmente en el mismo período, y se encontró un sesgo de entre dos y cuatro veces en las predicciones del aumento en la temperatura observada (Fyfe, et al., 2013). Este experimento llevó a proponer un hiato en la tendencia de calentamiento global en las últimas décadas, que después ha sido intensamente disputado y eventualmente descalificado en el marco de las discusiones del acuerdo climático (Rajaratnam, et al., 2015).

En resumen, es poco lo que sabemos acerca de los mecanismos que generan el ENOM, no lo suficiente en cualquier caso para ejecutar modelos determinísticos. Pero sí conocemos los efectos, amplitudes, recurrencias y teleconexiones actuales del ENOM, y podemos estudiar el registro geológico, con el fin de intentar discernir sus efectos en el pasado. A medida que nos internamos en el tiempo geológico, la incertidumbre en la datación precisa de los eventos y la dificultad de hacer correlaciones empíricas aumentan e impiden tener certeza sobre las relaciones de causalidad. Al adentrarse más en las profundidades del tiempo geológico y pasar a la escala de millones de años, cuando la temperatura del planeta (Zachos, et al., 2001) se parecía más a la que se espera al final del siglo XXI (Naciones Unidas, 2015), podríamos aprender más acerca de este fenómeno y sus impactos en Colombia, ya que los efectos locales y puntuales en el espacio y en el tiempo se difuminan $y$, aunque borrosa, aparece una visión empírica más robusta de las relaciones e interacciones entre el calentamiento global y el ENOM.

El Niño permanente del Plioceno, una visión global. El Plioceno, entre 2,6 y 5,3 millones de años, es el intervalo más reciente del pasado geológico con temperaturas sostenidas promedio más cercanas $\left(\sim 2,5\right.$ a $4{ }^{\circ} \mathrm{C}$, Zachos, et al., 2001) a las esperadas como resultado del cambio climático. De todo el pasado geológico, el Plioceno, además, presenta la configuración de placas, océanos y cadenas montañosas más similar a la actual (Scotese \& Golonka, 1997; Müller, et al., 2008), de modo que las corrientes oceánicas y atmosféricas tenían barreras y corredores parecidos, aunque no idénticos, a los de hoy en día. Por ello, es probable que este intervalo de tiempo sea el mejor parangón para prepararnos frente al comportamiento del clima del planeta en las décadas venideras (Wara, 2005).

La intrigante posibilidad de que el clima del Plioceno se haya caracterizado por un fenómeno de El Niño permanente fue propuesta por Molnar \& Cane $(2002$; 2007) al observar la coincidencia de condiciones climáticas del pasado con las que se han presentado durante eventos recientes de El Niño, observación coincidente con la de Philander \& Fedorov (2003). Durante el Plioceno el clima canadiense era más cálido y seco, el hielo en los grandes lagos en invierno era más delgado, y la región alrededor del Golfo de México y el sur del continente suramericano más fresca y húmeda. La India y el noreste australiano eran más secos que hoy, en tanto que Africa oriental era más húmeda. El contraste de temperaturas entre las aguas tropicales del Pacífico oriental y el occidental era mínimo, o inexistente. Todas estas observaciones paleoclimáticas se correlacionan positivamente con los fenómenos atmosféricos y oceánicos que se registran durante los actuales eventos de El Niño, en particular el de 1997 y, por ende, sugieren que el ENOM podría haber sido permanente durante el Plioceno. Wara (2005) llegó a conclusiones similares estudiando registros isotópicos de temperaturas marinas superficiales en conchas de foraminíferos en el Pacífico tropical. También en el Pacífico tropical, pero más al oriente y estudiando registros de alquenonas, Lawrence, et al. (2006) encontraron que la asimetría en temperaturas superficiales era prácticamente inexistente en el Plioceno, conclusión también apoyada en la revisión de diferentes indicadores climáticos (proxies) de Ravelo, et al. (2006). 
El registro paleoclimático terrestre del Plioceno en los trópicos es aún muy limitado, pues solo se tienen dos registros en Costa Rica y Etiopía, y ninguno a menos de $10^{\circ}$ del ecuador (Salzmann, et al., 2013). Sin embargo, el norte de los Andes, más seco y cálido durante eventos de El Niño de hoy, tiene un completo registro palinológico del Plioceno (van der Hammen, et al., 1973). Este registro documenta un cambio florístico entre $\sim 3$ y 6 millones de años, originalmente interpretado como resultado del levantamiento de la cordillera Oriental y el consiguiente enfriamiento por ganancia altitudinal (Helmens \& van der Hammen, 1994). Según esta hipótesis, durante el Plioceno temprano la Sabana de Bogotá habría estado localizada a una elevación de $\sim 500 \mathrm{~m}$ sobre el nivel del mar con una flora típica de tierras bajas tropicales. Un levantamiento de la superficie de $\sim 2$ kilómetros habría causado un cambio en la temperatura (entre 9 y $12{ }^{\circ} \mathrm{C}$, Hooghiemstra, et al., 2006) y la aparición de la flora de tierras altas, que hace 2,5 millones de años ya era la característica flora andina de altitud (van der Hammen, et al., 1973).

El cambio en temperaturas derivado del registro palinológico ha sido recientemente reevaluado a solo $\sim 3^{\circ} \mathrm{C}$ por Anderson, et al. (2015) utilizando biomarcadores en los lípidos de las membranas de bacterias presentes en los suelos. Estas cadenas de lípidos se encuentran preservadas en las mismas localidades de los estudios palinológicos y, con base en los cambios estructurales en las cadenas, se puede calcular una paleotemperatura, pues la complejidad de estas cadenas depende del $\mathrm{pH}$ y de la temperatura media anual de los suelos. Una vez el cambio en temperaturas es corregido por el clima global, este concordaría, no con un cambio de elevación, sino más probablemente con un estado de El Niño en el norte de los Andes (Pérez-Ángel \& Molnar, 2017) durante el Plioceno. Este último análisis se basa en la comparación del registro instrumental de las temperaturas de la Cordillera Oriental de 1961 a 2016 con las temperaturas de la superficie oceánica durante los eventos de El Niño de 1982-83, 1997-98 y 2015-16. Cuando las temperaturas de la superficie del océano Pacífico se incrementan en $4{ }^{\circ} \mathrm{C}$, las temperaturas en la cordillera Oriental lo hacen en $\sim 2{ }^{\circ} \mathrm{C}$. Este cambio, extrapolado a las condiciones climáticas del Plioceno es suficiente para explicar el cambio florístico sólo con un El Niño más intenso o más frecuente.

Sin embargo, en el registro palinológico no existe evidencia clara de condiciones más secas durante el Plioceno. Sólo el registro sedimentológico del delta del río Magdalena en la transición Mioceno-Plioceno marca la instalación de condiciones más secas en el norte de los Andes durante el Plioceno, consistente con la idea de un El Niño más intenso o frecuente (Molinares, et al., 2012). Aun así, es necesario emplear más marcadores independientes, por ejemplo, las rutas fotosintéticas de plantas $\mathrm{C} 3$ o $\mathrm{C} 4$, para evaluar las condiciones de precipitación en el norte de los Andes durante el Plioceno.
En resumen, existe evidencia suficiente que sugiere que en una época no muy distante del pasado geológico, cuando la configuración geográfica era similar a la de hoy y las temperaturas estaban dentro del rango esperado debido al cambio climático, el clima del planeta estaba dominado por un fenómeno de El Niño permanente. En el norte de los Andes dicho aumento en su intensidad o frecuencia parece haber estado acompañado por temperaturas más altas, pero aún no hay confirmación de que las precipitaciones fueran más bajas, como se esperaría si el fenómeno global tenía teleconexiones similares a las de hoy en día.

El Niño en Colombia. Los efectos del ENOM en el norte de los Andes se caracterizan actualmente por un déficit de precipitaciones y de los caudales y cargas medias mensuales de los ríos (Restrepo \& Kjerfve, 2000; Restrepo-López \& Torregroza, 2017), y una disminución en la humedad de los suelos y la actividad vegetal (Poveda, et al., 2001; Poveda, 2004) que, por consiguiente, disminuye el nivel de los embalses. Incluso existe una correlacion entre el índice de la oscilación meridional y los índices de malaria (Poveda \& Rojas, 1997), los cambios en la salinidad de ciénagas costeras debidos a la disminución de los caudales (Blanco, et al., 2007), los eventos de avulsión del río Magdalena (Morón, et al., 2017) y los glaciales tropicales (BedoyaSoto, et al., 2018).

La disminución de las precipitaciones durante un evento de El Niño es mayor precisamente en las áreas donde se encuentran las hidroeléctricas más importantes del país: Antioquia, Valle del Cauca, Cundinamarca y Tolima, así como en zonas de la Orinoquia (López, et al., 2013). La recurrencia moderna del fenómeno de El Niño está entre los 3 y 5 años o casi bienal (Wara, 2005), con registros más tempranos y marcados en los caudales de los ríos en el occidente de Colombia y más tardíos y menos marcados en los del este (Poveda, et al., 2001; Poveda, 2004). Un típico año de El Niño inicia con un déficit moderado en las precipitaciones entre junio y agosto y entre septiembre y noviembre, con la consecuente disminución en los caudales de los ríos. Durante los siguentes meses, especialmente entre diciembre y febrero, se presenta una disminución muy marcada en las precipitaciones y las descargas de los ríos, seguida por un comportamiento casi normal de la temporada de marzo a mayo (Poveda, et al., 2001).

El Niño que trajo el apagón de mayo de 1992 a febrero de 1993 está aún vivo en la memoria colectiva de los colombianos, cuando una marcada disminución en las lluvias colapsó la disponibilidad de los acueductos y el suministro energético causó cortes de entre nueve y 18 horas diarias en muchas partes del país (Unidad de Planeación Minero Energética - UPME, 2010), (http://www.elcolombiano. com/historico/hace_20_anos_colombia_sufrio_el_apagonJVEC_180119). El evento de El Niño del 2009 trajo una disminución en las precipitaciones que redujo el embalse agregado nacional a un $30 \%$ de su nivel en el mes de marzo del 2010 (UPME, 2010). 
Recursos hídricos y eventos de El Niño. Aunque el sistema interconectado nacional nunca ha tenido que soportar dos años seguidos con eventos significativos de El Niño, es fácil predecir cuáles serían sus consecuencias. En múltiples estudios se ha destacado la debilidad de nuestro sistema interconectado debido a su dependencia de la regularidad del sistema hídrico (Uribe, et al., 2014; UPME, 2015). En ningún escenario, sin embargo, se ha evaluado la posibilidad de que Colombia sufra dos o más años con fenómenos de El Niño consecutivos, como podría haber sido la norma en el Plioceno, o, incluso, a principios del siglo XX (Gergis \& Fowler, 2009).

Las proyecciones de los cambios en el régimen de precipitaciones en Colombia debidos al cambio climático, se limitan a extrapolar los datos instrumentales recientes (Macías \& Andrade, 2014), a partir de los cuales un leve descenso en las precipitaciones registrado instrumentalmente en los últimos $\sim 30$ años, se proyecta linealmente en el futuro. Esta lógica de predicción, con una disminución gradual en los aportes hídricos de 6 a $8 \%$, no tiene en cuenta que el régimen hidrológico del país es claramente no linear (Poveda, et al., 2001), ni tampoco la posibilidad de un ENOM permanente como lo sugieren los análisis paleoclimáticos globales (Molnar \& Cane, 2002; Wara, 2005; Molnar \& Cane, 2007), o locales del norte de los Andes (Molinares, et al., 2012; Pérez-Ángel \& Molnar, 2017). El componente paleoclimático en los escenarios de generación futura (UPME, 2015) brilla por su ausencia.

El sistema interconectado nacional depende de una temporada normal de marzo a mayo para iniciar la recuperación de los niveles de los embalses. Si llegase a faltar una sola de estas temporadas, la generación hídrica podría, sencillamente, no estar disponible por varios años.

Recursos térmicos y eventos de El Niño. A raíz del apagón de 1992 a 1993 el gobierno entendió la debilidad del sistema y diversificó la canasta energética (UPME, 2010) mediante la instalación de una mayor capacidad de generación térmica, fundamentalmente a partir de gas y combustibles líquidos. Como resultado, se agilizó la terminación de la hidroeléctrica del Guavio, se amplió la interconexión con Venezuela y se aseguró la inversión privada en generadoras térmicas con garantías de compra de energía a 15 y 20 años a precios fijos. Así, entraron en funcionamiento las centrales de Flores I (150 MW, 1994), Flores II (100 MW, 1996), Tebsa (750 MW, 1996), Termodorada (50 mW, 1997), Termovalle (150 MW, 1998), Termoemcali (220 MW, 1999) y Paipa-I (150 MW, 1999), con las que se logró esquivar el racionamiento eléctrico durante el evento de El Niño de 1997.

A partir de 1998 se ampliaron las redes de transmisión eléctrica del país y en el 2003 se inició la interconexión eléctrica con Ecuador. Apartir del 2006 se instauró el 'cargo por confiabilidad', con el cual se regulan las 'obligaciones de energía firme', un compromiso de los generadores para producir energía durante los periodos de condiciones críticas. El 'precio de escasez', cuando los precios de la bolsa exceden un umbral determinado, debía garantizar la solvencia económica de los generadores privados a la hora de cumplir con los compromisos establecidos en el 'cargo por confiabilidad'. Este esquema, sin embargo, no alcanzó a garantizar la solvencia de varios de los operadores y en el evento de El Niño del 2016 el gobierno tuvo que intervenir la operación de Termocandelaria, la cual argumentaba que no tenía disponibilidad porque el 'precio de escasez' no justificaba su operación (Figura 1), (http://www.eluniversal. com.co/colombia/proxima-terminar-la-intervencion-determocandelaria-225093). A pesar de las críticas del sector al cálculo del 'precio de escasez' (Associación Nacional de Empresas Generadoras - ANDEG, 2014), y la necesidad del gobierno de intervenir la operación de los generadores privados, el evento de El Niño del 2016 no llegó a doblegar el sistema interconectado nacional y se pudo esquivar, por muy poco, otro racionamiento energético.

En resumen, la respuesta al apagón de 1992-93 trajo un sistema descentralizado, interconectado, privado, pero regulado, que hasta ahora y con algunos inconvenientes, ha garantizado el suministro energético del país. El sector térmico, basado en gas ( $26 \%$ de la capacidad instalada, UPME, 2015) y en combustibles líquidos ha demostrado

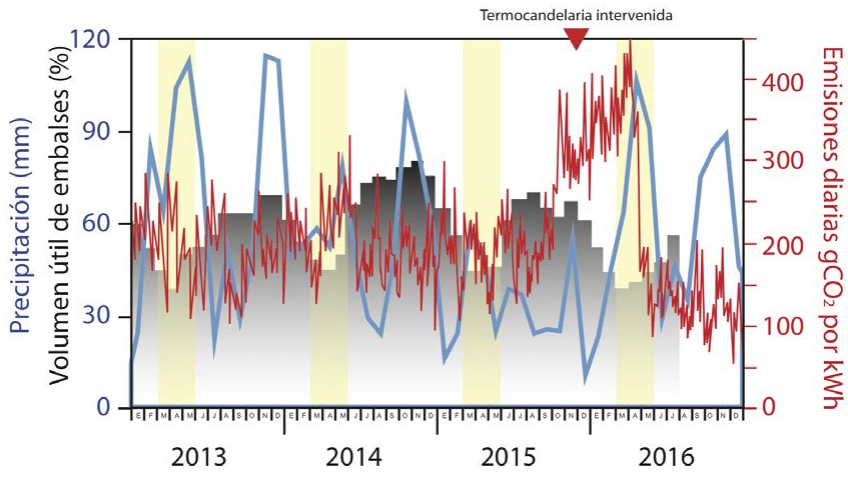

Figura 1. Figura 1. Ejemplo de cómo se comportan los niveles de los embalses ante escenarios de escasez de precipitación. El volumen útil de los embalses se expresa como porcentaje (barras grises hasta julio del 2016, UPME, 2016); las emisiones de CO2 (línea roja) se presentan como marcador de la generación térmica (http://informesanuales.xm.com.co/2017/SitePages/operacion/ Default.aspx), y se da el promedio de las precipitaciones en cuatro estaciones pluviométricas de la CAR (Fute en Soacha, La Ramada en Funza, Checua en Nemocón y Venecia en Facatativá) entre el 2013 y el 2016 (https://www.datos.gov.co/Ambiente-y-DesarrolloSostenible/Precipitaciones-Totales-Mensuales/mb4n-6m2g). La temporada de lluvias de marzo a mayo se resalta en amarillo, así como la fecha de inicio de la generación en la termoeléctrica Termocandelaria después de la intervención (ver texto). Aunque las precipitaciones graficadas no son representativas de todo el país, obsérvese cómo con solo dos temporadas lluviosas deficitarias, el nivel de los embalses se reduce a puntos críticos y las termoeléctricas deben entrar a operar a capacidad quemando gas importado. 
que puede llenar el vacío que deja la disminución en las precipitaciones durante un solo evento de El Niño. Pero el sistema interconectado nacional no ha experimentado todavía dos años consecutivos bajo las condiciones de El Niño.

Recursos no renovables y eventos de El Niño. Si suponemos que la generación hídrica puede ser golpeada por dos eventos intensos de El Niño consecutivos, podemos analizar entonces cuáles son las alternativas de generación. Aumentar la construcción de hidroeléctricas, sacrificando aun más nuestros ríos, sería multiplicar la incertidumbre, como ya lo anoté. La construcción de hidroeléctricas, además, tiene impactos ambientales y sociales en las cuencas locales y aguas abajo en importantes sistemas de humedales como la depresión momposina (Angarita, et al., 2018). Descarto, sin mayor discusión, la opción nuclear, pues Colombia está muy lejos, tecnológica y políticamente, de consi-derar una opción de este tipo. Después de la fusión del reactor de Fukushima, Japón, de forma muy pragmática (Friedel, et al., 2011), parece haber abandonado la opción nuclear a favor del carbón (Normile, 2018). Alemania está en el mismo proceso, pero a favor de los renovables. Los recursos eólicos, solares y geotérmicos, aunque ampliamente discutidos y ponderados en círculos políticos y ambientalistas colombianos, han tenido una inversión prácticamente nula en investigación y desarrollo (una fracción infinitesimal del 0,27 \% del PIB, Colciencias, 2017). De modo que a corto y mediano plazo los renovables no son una opción realista para llenar el probable déficit de generación hídrica y la satisfacción de las dos terceras partes del consumo del país. Para com-parar la magnitud de la inversión necesaria, y según datos de la Agencia de Energías Renovables de Alemania, el energiewende o la transformación energética requerirá una inversión más de 200 mil millones de euros (2010-2020), un compromiso político y económico de toda la sociedad, y varias décadas para alcanzar una meta de apenas el $47 \%$ de la electricidad y $28 \%$ de la energía total alemana. Las inversiones de Colombia están muy, muy lejos, de estas cifras.

Así las cosas, las opciones colombianas para garantizar la generación de energía eléctrica en un escenario de cambio climático con un ENOM más intenso o frecuente, se reducen a aprovechar los recursos no renovables. Como explico a continuación, la ventana de autoabastecimiento de gas y petróleo es de menos de cinco años, lo que deja al carbón como la única alternativa viable para mantener la independencia energética.

Los recursos no renovables en Colombia. Colombia es un país geológicamente muy diverso debido a la acreción secuencial de terrenos geológicos a lo largo de su borde occidental (Cediel, et al., 2003; Spikings, et al., 2015). Hace aproximadamente 90 a 100 millones de años un período prolongado de anoxia oceánica (escasez de oxígeno disuelto en la interfase agua-sedimento) causó la preservación de espesores importantes de rocas ricas en materia orgánica a lo largo del todo el borde noroccidental de Suramérica
(Moreno-Sánchez \& Pardo-Trujillo, 2003). Estas rocas de grano fino son característicamente negras (shale negro), y se conocen en Colombia y Venezuela como Formación La Luna (Villamil, 2003). Hace aproximadamente 80 millones de años, arcos de islas intraoceánicas colisionaron con el borde occidental de Colombia, barriendo de sur a norte (Villamil, 1999; Weber, et al., 2009; Cardona, et al., 2012), causando deformación y metamorfismo y destruyendo la materia orgánica necesaria para la generación de hidrocarburos. Más al oriente, estas secuencias se preservaron en cuencas internas protegidas de la deformación, que hoy son productivas (Villamil, 2003; Escalona \& Mann, 2006). Más tarde, hace aproximadamente 65 a 55 millones de años, el mar que había inundado gran parte del actual territorio colombiano, se retiró paulatinamente dejando grandes extensiones de pantanos tropicales donde se acumularon enormes espesores de materia orgánica vegetal que después se convirtieron en carbón (Bayona, et al., 2011). Así, la cordillera Oriental, el piedemonte y la cuenca de los ríos Cesar y Ranchería, son territorios ricos en carbón, con reservas de miles de millones de toneladas.

Por definición, los recursos no renovables se agotan, ejemplo de ello es la cuenca del Valle Medio del Magdalena, donde se han extraído más de 3.000 millones de barriles de hidrocarburos (Cediel, 2011), y donde, recientemente, el campo Cira Infantas cumplió 100 años de explotación (https://www.larepublica.co/economia/los-hitos-de-los-100anos-de-extraccion-de-petroleo-en-colombia-2717278). Esta es una cuenca madura para los recursos convencionales, es decir, los esfuerzos exploratorios han dado frutos y muchos campos ya se han agotado y ahora están en proceso de recuperación asistida. En el otro extremo están las cuencas frontera, donde aun no hay hallazgos pero sí exploración activa, y se espera encontrar yacimientos con potencial comercial. Este podría ser el ejemplo del Caribe colombiano, donde varias empresas han invertido enormes recursos financieros y tecnológicos en la búsqueda de hidrocarburos. Sin embargo, el sistema petrolífero podría, sencillamente, estar ausente. Los ejemplos recientes de descubrimientos de gas sugieren que se trata de acumulaciones biogénicas, es decir, por descomposición de materia orgánica y no por el proceso de su maduración para formar hidrocarburos (http:// www.rigzone.com/news/oil_gas/a/136194/Petrobras_Finds_ Gas_at_Orca1_Well_in_Colombias_Offshore_Tayrona Block). Sin tener en cuenta una posible revolución de recursos no convencionales, Colombia está hoy en el medio del espectro, con cuencas maduras, aún en producción, pero en franco declive, y cuencas frontera, donde aún no se han probado acumulación de hidrocarburos con potencial comerciales. Por ello, el gobierno ha reconocido que en las condiciones actuales, la autosuficiencia petrolera del país es de menos de cinco años y la gasífera, incluso menor (UPME, 2015). No obstante, el cálculo de la autosuficiencia es complejo, ya que los incrementos en el precio del barril hacen que las reservas más caras, más inaccesibles, se 
vuelvan comerciales y rentables a medida que sube el precio. El efecto contrario ocurre con las caídas en los precios, lo cual conlleva la contracción de las reservas. Sea como sea, nos estamos quedando sin gas y sin petróleo.

\section{El carbón como alternativa}

Colombia, en cambio tiene carbón, mucho carbón, y de muy buena calidad, típicamente con contenidos de azufre de menos del 1\% (Beltrán, 1974). Por eso el país lleva décadas exportando a Europa millones de toneladas de carbón térmico al año ( $\sim 90$ millones de toneladas, UPME, 2013), mayoritariamente de la cuenca del Cesar-Ranchería. Esa cantidad de carbón que exportamos es suficiente para llevar electricidad a 30 millones de hogares en países desarrollados en un año o, dicho de otra manera, con la producción de un solo año se puede alimentar una planta térmica de $600 \mathrm{Mw}$ durante cerca de 60 años operando a capacidad, suponiendo un $38 \%$ de eficiencia y una disponibilidad del $75 \%$. Colombia, entonces, exporta mucho carbón, pero produce menos del $5 \%$ de su electricidad a partir de este mineral, y el crecimiento que se proyecta es de menos del $12,5 \%$ de la capacidad instalada para el 2028 (UPME, 2015). El resto de la generación térmica es a gas, con diesel y otros combustibles líquidos que, como sabemos, se están agotando en nuestras cuencas.

Incluso en una situación de precipitaciones normales, sin considerar cambios en el ENOM, Colombia ya está importando gas licuado (la forma costosa del gas) y combustibles líquidos, ambos amarrados al volátil precio del barril de petróleo (https://www.dinero.com/economia/ articulo/gas-en-colombia-se-importa-o-se-produce-seguncanacol/243683). Es contradictorio que un país tan rico en energía (en forma de carbón) tenga que construir plantas regasificadoras para poder importar gas licuado de los Estados Unidos (país que, como dije arriba, ya se retiró del acuerdo de París), para quemar en su sector térmico. Si se da un ENOM más intenso o más frecuente, el sector térmico deberá importar grandes cantidades de gas licuado, y el país pasaría a ser un importador neto de energía, sin siquiera llegar a cubrir toda su demanda. El gas importado podría llegar a costar más del triple del precio del gas nacional, sin tener en cuenta los costos de transporte y regasificación (UPME, 2015).

Enfrentado a una disyuntiva comparable, Japón ha decidido quemar carbón, no gas. Una razón pueden ser los costos, ya que una vez que se decide importar gas, hay que hacerlo en su forma licuada, lo que lo hace más costoso. Otra razón de mayor peso puede ser estratégica. El carbón es fácilmente apilable como reserva a largo plazo en los patios traseros de las plantas térmicas, y a muy bajo costo. La posibilidad de apilamiento estratégico barato a largo o muy largo plazo es una ventaja que solo tiene el carbón. No se puede decir lo mismo de ninguna otra fuente de energía.

Aprovechando sus reservas de carbón, Colombia podría suplir la totalidad de su consumo de energía eléctrica y convertirse en un exportador neto de electricidad a países vecinos. Las nuevas tecnologías aumentan la eficiencia de las plantas (Schrag, 2009), y podrían hacerlo más limpio (https:// www.bbc.com/news/science-environment-35659947), (https://www.bbc.com/news/science-environment-39032748) que el gas (Álvarez, et al., 2018) procesándolo en plantas de ciclo ultra-supercrítico, donde las presiones y las temperaturas del vapor exceden los 300 bar y los $600^{\circ} \mathrm{C}$ (http://www. powerengineeringint.com/articles/print/volume-25/issue-3/ features/critical-thinking.html).

La minería de carbón a cielo abierto ciertamente tiene impactos ambientales negativos. La cuenca del CesarRanchería es la principal productora de carbón del país, y una de las más grandes del mundo. Dichos impactos incluyen cambios en la calidad del aire en las comunidades vecinas a los tajos de minería, ruido, vibraciones, impactos visuales, y otros. Además, la huella de los tajos no solo implica el tajo mismo, sino también los depósitos de material estéril, las vías de acceso y la infraestructura de transporte, manejo y embarque. Así, el valle del Cesar-Ranchería, en particular en su zona más septentrional, donde es más angosto, es una zona casi totalmente intervenida por la minería. Desde el piedemonte de la serranía del Perijá al de la Sierra Nevada de Santa Marta existen depósitos de material estéril y tajos de minería. La zona minera se ve interrumpida solo por el todavía bello río Ranchería, su acuífero y su bosque de galería, protegido dentro de la zona minera.

Sin embargo, y apelando al pragmatismo, el impacto (y las compensaciones) de la minería del carbón en la cuenca del Cesar-Ranchería ya están causadas. La minería a cieloabierto inició a gran escala en la década de los años 80 y ha continuado de manera ininterrumpida desde entonces. Cerrar las minas, o dejar que lentamente se ahoguen con la previsible caída de la demanda europea, no arreglaría los impactos, y de hecho podría agravarlos, ya que no habría un ente responsable por la administración y manejo de estas áreas. La reducción del consumo en algunos países que tradicionalmente han sido grandes consumidores de carbón, como el Reino Unido, ya están contrayendo los mercados del carbón colombiano (https://www.nytimes.com/2017/04/21/ world/europe/britain-burning-coal-electricity.html). Tales reducciones podrían ser suplidas por el consumo interno para la generación de electricidad. Permitir el cierre de las minas de carbón colombianas, mientras importamos gas licuado y combustibles líquidos, sería un contrasentido. Le estaríamos dando la espalda al único recurso que podría asegurar nuestra independencia y seguridad energética.

\section{Conclusiones}

En este ensayo presento una mirada pragmática sobre la encrucijada energética en la que se encuentra Colombia. Mientras que es claramente preferible tener una economía verde donde toda la energía del país provenga de energías limpias y amigables con el medio ambiente, no tenemos muchas alternativas diferentes al carbón. Décadas de inversiones mínimas o nulas en energías renovables, donde 
Colombia podría ser una potencia, como la eólica y solar en la costa Caribe, o la geotérmica en el occidente colombiano, hace que el camino por recorrer se aún muy largo. Tan largo de hecho, que los efectos del cambio climático podrían llegar antes que la renovación energética.

Esta mirada podría ser más despreocupada, podríamos, por ejemplo, sentarnos a esperar que en vez de una disminución el cambio climático traiga un aumento en las precipitaciones (como en los años de eventos de $\mathrm{La}$ Niña), en cuyo caso la generación hídrica estaría de plácemes a corto plazo. También podríamos esperar que los esfuerzos exploratorios en el Caribe colombiano sean exitosos y traigan nuevas y abundantes reservas de gas económicamente explotables. Sin embargo, la preparación frente a situaciones desconocidas debe considerar por lo menos un escenario con la peor combinación de factores, que en Colombia incluye tres elementos fundamentales: 1) eventos de ENOM con déficits consecutivos (por lo menos dos) en las temporadas lluviosas; 2) una matriz energética basada en la hidrogeneración, y 3) respaldada por gas y combustibles líquidos importados. Infortunadamente, de los tres factores arriba mencionados, Colombia ya tiene los dos últimos sólidamente establecidos en su infraestructura energética. Recordemos que más de dos terceras partes de nuestra generación es hidroeléctrica, y que nuestras plantas térmicas queman principalmente gas, no carbón. Un ENOM más intenso, o simplemente dos eventos consecutivos de este tipo, sería el tercer factor detonante de una crisis del sector energético. Colombia entonces pasaría a depender del gas licuado y los combustibles líquidos importados, o de la interconexión eléctrica con países vecinos (Ecuador y Venezuela). En cualquiera de los casos, y con nuestras termoeléctricas quemando gas y no carbón, Colombia pierde independencia y confiabilidad en el suministro energético.

Mientras nos preparamos para los efectos desconocidos del ineludible calentamiento global, debemos seguir aprendiendo más acerca del régimen climático en el norte de los Andes durante el Plioceno. Todavía es muy poco lo que sabemos, pero puede potencialmente ser la clave para planear mejor nuestras alternativas de matriz energética en el mediano y largo plazo. Debemos también, como lo indica el documento de las partes COP21, eliminar o reversar las tendencias de deforestación actuales. Aunque no se ha calculado todavía, solo manteniendo nuestros bosques tropicales intactos y generando toda nuestra electricidad de carbón, podríamos mantener nuestras emisiones netas de $\mathrm{CO}_{2}$ en cero.

\section{Agradecimientos}

A J. H. Escobar, al Instituto de Desarrollo Sostenible de la Universidad del Norte y A. I. Cadena de la Universidad de Los Andes, por fomentar seminarios sobre el tema. A M.L. Rodríguez-Puerto por las correcciones idiomáticas. A L. Pérez-Ángel por sus comentarios sobre el clima del Plioceno en los trópicos. A los revisores y al editor por sus sugerencias y comentarios para mejorar el manuscrito. Las opiniones aquí expresadas, así como cualquier error factual o de interpretación, son responsabilidad del autor.

\section{Referencias}

Adams, J. B., Mann, M. E., Ammann, C. M. 2003. Proxy evidence for an El Niño-like response to volcanic forcing. Nature. 426 (6964): 274

Álvarez, R. A., Zavala-Araiza, D., Lyon, D. R., Allen, D. T., Barkley, Z. R., Brandt, A. R., Davis, K. J., Herndon, S. C., Jacob, D. J., Karion, A., et al. 2018. Assessment of methane emissions from the US oil and gas supply chain. Science. 361 (6398): 186-188. doi: 10.1126/science. aar7204

Asociación Nacional de Empresas Generadoras - ANDEG. 2014. Un análisis del precio de escasez. ANDEG. Bogotá: 28.

Anderson, V. J., Saylor, J. E., Shanahan, T. M., Horton, B. K. 2015. Paleoelevation records from lipid biomarkers: Application to the tropical Andes: GSA Bulletin. 127 (11-12): 1604-1616.

Angarita, H., Wickel, A. J., Sieber, J., Chavarro, J., MaldonadoOcampo, J. A., Herrera-R, G. A., Delgado, J., Purkey, D. 2018. Basin-scale impacts of hydropower development on the Mompós Depression wetlands, Colombia. Hydrology and Earth System Sciences. 22 (5): 2839.

Bathiany, S., Dakos, V., Scheffer, M., Lenton, T. M. 2018. Climate models predict increasing temperature variability in poor countries. Science advances. 4 (5): eaar5809. doi: 10.1126/sciadv.aar5809

Baumert, K. A., Herzog, T., Pershing, J. 2005. Navigating the Numbers, Greenhouse Gas Data and International Climate Policy, U.S.A. World Resources Institute. Washington DC, World Resources Institute: 122.

Bayona, G. A., Montes, C., Cardona, A., Jaramillo, C., Ojeda, G., Valencia, V., Ayala-Calvo, R. C. 2011. Intraplate subsidence and basin filling adjacent to an oceanic arccontinent collision: A case from the southern CaribbeanSouth America plate margin. Basin Research. 23: 403-422.

Bedoya-Soto, J. M., Poveda, G., Trenberth, K. E., VélezUpegui, J. J. 2018. Interannual hydroclimatic variability and the 2009-2011 extreme ENSO phases in Colombia: From Andean glaciers to Caribbean lowlands. Theoretical and Applied Climatology. https://doi.org/10.1007/s00704018-2452-2:1-14.

Beltrán, E. 1974. Carbones de Colombia. Bogotá, Ministerio de Minas y Petróleos: 254.

Blanco, J. A., Barandica, J. C. N., Viloria, E. A. 2007. ENSO and the rise and fall of a tilapia fishery in northern Colombia. Fisheries Research. 88 (1-3): 100-108.

Bove, M. C., O’Brien, J. J., Eisner, J. B., Landsea, C. W., Niu, X. 1998. Effect of El Niño on US landfalling hurricanes, revisited. Bulletin of the American Meteorological Society. 79 (11): 2477-2482.

Cai, W., Borlace, S., Lengaigne, M., Van Rensch, P., Collins, M., Vecchi, G., Timmermann, A., Santoso, A., McPhaden, M. J., Wu, L. 2014. Increasing frequency of extreme El Niño events due to greenhouse warming. Nature climate change. 4 (2): 111.

Cai, W., Santoso, A., Wang, G., Yeh, S.-W., An, S.-I., Cobb, K. M., Collins, M., Guilyardi, E., Jin, F.-F., Kug, J.-S., et al. 2015. ENSO and greenhouse warming. Nature Climate Change. 5 (9): 849. 
Cardona, A., Montes, C., Ayala, C., Bustamante, C., Hoyos, N., Montenegro, O., Ojeda, C., Niño, H., Ramírez, V., Valencia, V., Rincón, D., Vervoort, J. D., Zapata, S. 2012. From arc-continent collision to continuous convergence, clues from Paleogene conglomerates along the southern Caribbean-South America plate boundary. Tectonophysics. 580: 58-87.

Cediel, F. 2011. Petroleum Geology of Colombia. Geology and Hydrocarbon Potential. Regional Geology of Colombia, Medellín. p. 225.

Cediel, F., Shaw R.P., Cáceres, C. 2003. Tectonic assembly of the northern Andean Block. AAPG Memoir. 79: 815-848.

Cobb, K. M., Charles, C. D., Cheng, H., Edwards, R. L. 2003. El Niño/Southern Oscillation and tropical Pacific climate during the last millennium. Nature. 424 (6946): 271.

Colciencias. 2017. Boletín estadístico No 5: Departamento Administrativo de Ciencia, Tecnología e Innovación.

Collins, M., An, S.-I., Cai, W., Ganachaud, A., Guilyardi, E., Jin, F.-F., Jochum, M., Lengaigne, M., Power, S., Timmermann, A. 2010. The impact of global warming on the tropical Pacific Ocean and El Niño. Nature Geoscience. 3 (6): 391.

Dai, A. \& Wigley, T. 2000. Global patterns of ENSO-induced precipitation. Geophysical Research Letters. 27 (9): 1283 1286.

Donnelly, J. P. \& Woodruff, J. D. 2007. Intense hurricane activity over the past 5,000 years controlled by El Niño and the West African monsoon. Nature. 447 (7143): 465.

Escalona, A. \& Mann, P. 2006. An overview of the petroleum system of Maracaibo Basin. AAPG Bulletin. 90 (4): 657-678.

Friedel, J., Bard, P., Bernard, P., Cara, M., Courtillot, V., Dercourt, J., Jaupart, C., Le Pichon, X., Madariaga, R., Mantagner, J., Pecker, A., Poirier, J., Salencon, J., Schiendele, F., Tapponnier, P. 2011. Interim Report by an ad hoc working party of the Academie des Sciences. Academie des Sciences: 32 .

Fyfe, J. C., Gillett, N. P., Zwiers, F. W. 2013. Overestimated global warming over the past 20 years. Nature Climate Change. 3 (9): 767.

Gergis, J. L. \& Fowler, A. M. 2009. A history of ENSO events since A.D. 1525: Implications for future climate change. Climatic Change. 92: 343-387.

Helmens, K. \& van der Hammen, T. 1994. The Pliocene and Quaternary of the high plain of Bogotá (Colombia): A history of tectonic uplift, basin development and climatic change. Quaternary International. 21: 41-61.

Hooghiemstra, H., Wijninga, V., Cleef, A. M. 2006. The paleobotanical record of Colombia: Implications for biogeography and biodiversity. Annals of the Missouri Botanical Garden. 93: 297-324.

Kreft, S., Eckstein, L., Kerestan, C., Hagen, U. 2015. Global Climate Risk Index 2015: Who Suffers Most From Extreme Weather Events? Weather-related Loss Events in 2013 and 1994 to 2013. Germanwatch e.V., Bonn: 32.

Lawrence, K. T., Liu, Z., Herbert, T. D. 2006. Evolution of the eastern tropical Pacific through Plio-Pleistocene glaciation. Science. 312 (5770): 79-83.

López, J. A., Medina, G., Domínguez, E., Uribe, E., Zapata, W., Arango, S., Martínez, J. F., Correa, C. 2013. Estudio para determinar la vulnerabilidad y las opciones de adaptación del sector energético colombiano frente al cambio climático. Unidad de Planeación Minero-Energética - UPME. Bogota: 416.

Macías, A. M. \& Andrade, J. 2014. Estudio de generación eléctrica bajo escenario de cambio climático. Unidad de Planeación Minero-Energética - UPME: Bogota: 106.

Millar, R. J., Fuglestvedt, J. S., Friedlingstein, P., Rogelj, J., Grubb, M. J., Matthews, H. D., Skeie, R. B., Forster, P. M., Frame, D. J., Allen, M. R. 2017. Emission budgets and pathways consistent with limiting warming to $1.5 \mathrm{C}$. Nature Geoscience. 10: 741-748.

Molinares, C., Martínez, J., Fiorini, F., Escobar, J., Jaramillo, C. 2012. Paleoenvironmental reconstruction for the lower Pliocene Arroyo Piedras section (Tubará-Colombia): Implications for the Magdalena River-paleodelta's dynamic. Journal of South American Earth Sciences. 39: 170-183.

Molnar, P. \& Cane, M. A. 2002. El Niño's tropical climate and teleconnections as a blueprint for pre-Ice Age climates. Paleoceanography. 17 (2): 11.

Molnar, P. \& Cane M.A. 2007. Early Pliocene (pre-Ice Age) El Niño-like global climate; which El Niño? Geosphere. 3 (5): 337-365.

Moreno-Sánchez, M. \& Pardo-Trujillo, A. 2003. Stratigraphical and sedimentological constraints on western Colombia: Implications on the evolution of the Caribbean plate. En: Bartolini, C., Buffler, R., and Blickwede, J., editors. The Circum-Gulf of México and the Caribbean: Hydrocarbon habitats, basin formation, and plate tectonics. 79: 891-924.

Morón, S., Amos, K., Edmonds, D. A., Payenberg, T., Sun, X., Thyer, M. 2017. Avulsion triggering by El Niño-Southern Oscillation and tectonic forcing: The case of the tropical Magdalena River, Colombia. GSA Bulletin. 129 (9-10): 1300-1313.

Müller, R. D., Sdrolias, M., Gaina, C., Roest, W. R. 2008. Age, spreading rates, and spreading asymmetry of the world's ocean crust. Geochemistry, Geophysics, Geosystems. 9 (4): 19 .

Normile, D. 2018. Bucking global trends, Japan again embraces coal power. Science. 360 (6388): 476-477.

Pérez-Ángel, L. C. \& Molnar, P. 2017. Sea Surface Temperatures in the Eastern Equatorial Pacific and Surface Temperatures in the Eastern Cordillera of Colombia During El Niño: Implications for Pliocene Conditions. Paleoceanography. 32 (11): 1309-1314.

Philander, S. G. \& Fedorov, A. V. 2003. Role of tropics in changing the response to Milankovich forcing some three million years ago. Paleoceanography. 18 (2): 23.

Poveda, G. 2004. La hidroclimatología de Colombia: una síntesis desde la escala inter-decadal hasta la escala diurna. Rev. Acad. Colomb. Cienc. Ex. Fis. Nat. 28 (107): 201-222.

Poveda, G., Jaramillo, A., Gil, M. M., Quiceno, N., Mantilla, R. I. 2001. Seasonality in ENSO-related precipitation, river discharges, soil moisture, and vegetation index in Colombia. Water resources research. 37 (8): 2169-2178.

Poveda, G. \& Rojas, W. 1997. Evidencias de la asociación entre brotes epidémicos de malaria en Colombia y el fenómeno El Niño-Oscilación del Sur. Rev. Acad. Colomb. Cienc. Ex. Fis. Nat. 21 (81): 421-429.

Rajaratnam, B., Romano, J., Tsiang, M., Diffenbaugh, N. S. 2015. Debunking the climate hiatus. Climatic Change. 133 (2): $129-140$. 
Ravelo, A. C., Dekens, P. S., McCarthy, M. 2006. Evidence for El Niño-like conditions during the Pliocene. Gsa Today. 16 (3): 4.

Rein, B., Luckge, A., Reinhardt, L., Sirocko, F., Wolf, A., Dullo, W. C. 2005. El Niño variability off Perú during the last 20,000 years. Paleoceanography. 20 (4): 17.

Restrepo, J. \& Kjerfve, B. 2000. Magdalena river: Interannual variability (1975-1995) and revised water discharge and sediment load estimates. Journal of hydrology. 235 (1-2): 137-149.

Restrepo-López, J. C. \& Torregroza, A. C. 2017. Suspended sediment load in northwestern South America (Colombia): A new view on variability and fluxes into the Caribbean Sea. Journal of South American Earth Sciences. 80: 340-352.

Rogelj, J., Luderer, G., Pietzcker, R. C., Kriegler, E., Schae, M., Krey, V., Riahi, K. 2015. Energy system transformations for limiting end-of-century warming to below $1.5{ }^{\circ} \mathrm{C}$. Nature Climate Change. 5 (6): 519, 527.

Ropelewski, C. F. \& Halpert, M. S. 1986. North American precipitation and temperature patterns associated with the El Niño/Southern Oscillation (ENSO). Monthly Weather Review. 114 (12): 2352-2362.

Salzmann, U., A. M., Dolan, A. M., Haywood, W. L., Chan, J., Voss, D. J., Hill, A., Abe-Ouchi, B., Otto-Bliesner, F. J., Bragg, M. A., Chandler, C., Contoux, H. J., Dowsett, A., Jost, Y., Kamae, G., Lohmann, D. J., Lunt, S. J., Pickering, M. J., Pound, G., Ramstein, N. A., Rosenbloom, L., Sohl, C., Stepanek, H., Ueda, Z., Zhang, Z. 2013. Challenges in quantifying Pliocene terrestrial warming revealed by data-model discord. Nature Climate Change. 3: 969-974.

Schrag, D. 2009. Coal as a low-carbon fuel? Nature Geoscience. 2 (12): 818.

Scotese, C. R. \& Golonka, J. 1997. Paleogeographic atlas, PALEOMAP Project, University of Texas at Arlington: 35.

Spikings, R. A., Cochran, R., Villagómez, D., van der Lelij, R., Vallejo, C., Winker, W., Beate, B. 2015. The geological history of northwestern South America: From Pangaea to the early collision of the Caribbean Large Igneous Province (290-75 Ma). Gondwana Research. 27: 96-139.

Timmermann, A., Oberhuber, J., Bacher, A., Esch, M., Latif, M., Roeckner, E. 1999. Increased El Niño frequency in a climate model forced by future greenhouse warming. Nature. 398 (6729): 694.

Tollefson, J. 2017. Trump pulls United States out of Paris climate agreement. Nature News. 546 (7657): 198.

Naciones Unidas. 2015. Convención Marco sobre el Cambio Climático: Conferencia de las Partes, 21er período de sesiones: Naciones Unidas. París: 40.
Unidad de Planeación Minero-Energética - UPME. 2010. Boletín Estadístico de Minas y Energía 1990-2010. Bogota: 248.

Unidad de Planeación Minero-Energética - UPME. 2013. Boletín Estadístico de Minas y Energía 2000-2013. Bogotá: 263.

Unidad de Planeación Minero-Energética - UPME. 2015. Plan Energético Nacional Colombia: Ideario Energético 2050. Bogotá:183.

Unidad de Planeación Minero-Energética - UPME. 2016. Boletín Estadístico de Minas y Energía, 2012-2016: Bogotá: 200.

Uribe, E., Cruz, G., Arango, S., Ramírez, C., León, I., Domínguez, E., García, L. C., Reyes, J. M. 2014. Definir estrategias del mapa de ruta para la adaptación del sector energético frente al cambio climático: UPME, Unidad de Planeación Minero Energética::203.

van der Hammen, T., Werner, J., van Dommelen, H. 1973. Palynological record of the upheaval of the Northern Andes: A study of the Pliocene and Lower Quaternary of the Colombian Eastern Cordillera and the early evolution of its High-Andean biota. Review of Palaeobotany and Palynology. 16 (1-2): 1-122.

van Oldenborgh, G. J., Philip, S., Collins, M. 2005. El Niño in a changing climate: A multi-model study. Ocean Science. 1 (2): 81-95.

Villamil, T. 1999. Campanian-Miocene tectonostratigraphy, depocenter evolution and basin development of Colombia and western Venezuela. Palaeogeography, Palaeoclimatology, Palaeoecology. 153 (1): 239-275.

Villamil, T. 2003. Regional hydrocarbon systems of Colombia and western Venezuela; their origin, potential, and exploration. AAPG Memoir. 79: 697-734.

Wara, M. W. 2005. Permanent El Niño-Like Conditions During the Pliocene Warm Period. Science. 309 (758): 758-761.

Weber, M., Cardona, A., Paniagua, F., Cordani, U., Sepúlveda, L., Wilson, R. 2009. The Cabo de la Vela Mafic-Ultramafic Complex, Northeastern Colombian Caribbean region: A record of multistage evolution of a Late Cretaceous intra-oceanic arc. Geological Society, London, Special Publications. 328 (1): 549-568.

Woodall, J. M. 2011. Physics for Future Presidents. New York, W. W. Norton \& Company: 361.

Yeh, S.-W., Kug, J.-S., Dewitte, B., Kwon, M.-H., Kirtman, B. P., Jin, F.-F. 2009. El Niño in a changing climate. Nature. 461 (7263): 511.

Zachos, J., Pagani, M., Sloan, L., Thomas, E., Billups, K. 2001. Trends, rhythms, and aberrations in global climate $65 \mathrm{Ma}$ to present. Science. 292 (5517): 686-693. 